\title{
Measurement of Corneal Neovascularisation
} with the Use of Image Processing Techniques

\author{
K. Murawski ${ }^{a *}$, D. Biąas ${ }^{b}$ And M. RęKas ${ }^{b}$ \\ ${ }^{a}$ Military University of Technology, Institute of Teleinformatics and Automatics, \\ S. Kaliskiego 2, 00-908 Warsaw, Poland \\ ${ }^{b}$ Military Institute of Medicine, Ophthalmology Department, Szaserów 128, 04-141 Warsaw, Poland \\ (Received May 12, 2014; in final form May 11, 2015)
}

\begin{abstract}
The aim of the article is to present the developed technique to measure the degree of neovascularisation of the cornea image in visible light. The study used an image sensor attached to a slit lamp. The proposed technique consists in performing segmentation of the color image to enhance blood vessels and setting values of the degree of cornea neovascularisation. Comparison of the indicator before and after treatment of the cornea can objectively assess the effectiveness of the adopted method of treatment.
\end{abstract}

DOI: $10.12693 /$ APhysPolA.127.1732

PACS: 87.57.-s, 87.57.N-, 87.57.nm

\section{Introduction}

The paper presents application of image segmentation technique as a tool to measurement of corneal neovascularisation. Image segmentation is performed to determine the position of the corneal blood vessels, measuring their area and finally measuring the degree of neovascularisation. The method is based on an analysis of the image of the cornea obtained in the visible light range.

The cornea is the front part of the fibrous membrane (tunica fibrosa bulbi) of the eye, which is the outer layer and the first critical element of the optical system which the eye is (central corneal refractive power of $+43.0 \mathrm{D}[1,2])$. In physiological conditions, in humans and in majority of animal species, the cornea, apart from a small peripheral area of the limbus, is avascular. However, in many clinical and experimental conditions blood vessels shows invasiveness and comes to corneal neovascularisation [3]. The new creation of blood vessels in the cornea is clinically an extremely important issue. Neovascularisation facilitates the formation of edema and lipid deposits, which leads to impaired visual acuity and also significantly worsens the corneal graft prognosis.

From a medical point of view it is important to develop an indicator and methods for measuring the degree of corneal neovascularisation. Such an indicator will assess the results of the treatment method used and also objectively carry out diagnostic imaging.

This solution is based on image analysis, done with a CCD camera attached to a slit lamp, Fig. 1a. Attempts to use these solutions have already been taken [4]. However, use of imaging concern mostly blood vessels in the retina [5-7], Fig. 1b, eyelids [8, 9], or the pupil [10-14].

Few studies have been published showing the use of imaging techniques to study neovascularisation of the

${ }^{*}$ corresponding author; e-mail: k.murawski@ita.wat.edu.pl
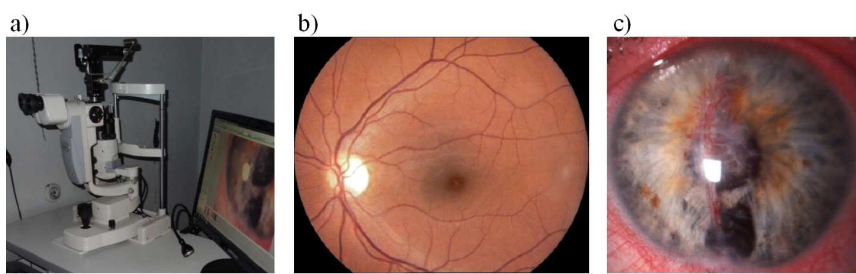

Fig. 1. Station for acquiring images: slit lamp with sensor (a), blood vessels of the retina (b), blood vessels of the cornea (c).

cornea [15]. This is undoubtedly due to the difficulty of obtaining good quality images of corneal blood vessels and problems during their processing. Due to the transparent character of the cornea, blood vessel imaging is difficult, Fig. 1c. As a result of the image processing technique used for image segmentation of blood vessels of the retina, in the case of the cornea, has limited application. In clinical practice, retinal vascular imaging is carried out using a fluorescent angiography FA [16]. This method is based on the clinical use of the fluorescence effect, i.e. the light emission of a particular wavelength (in a time less than $10^{-8} \mathrm{~s}$ ) under light excitation. This involves the movement of electrons to the highest orbits. The return to the state prior to excitation indicates the emission of quantum radiation of a lower energy and thereby a longer wavelength. A chemical compound - the contrast $\left(\mathrm{C}_{20} \mathrm{H}_{12} \mathrm{O}_{5} \mathrm{Na}\right.$ - fluorescein $)$ - is administered through the basilic vein into the bloodstream getting into the blood vessels of the eye. It can absorb electromagnetic energy with the transition to a higher, unstable energy level - singlet. In the case of the sodium salt of fluorescein the absorption peak at a $\mathrm{pH}$ of 7.4 appears at a $\lambda_{\mathrm{A}}$ wavelength of $465 \mathrm{~nm}$ to $490 \mathrm{~nm}$ (blue light) and the $\lambda_{\mathrm{E}}$ emission peak from $520 \mathrm{~nm}$ to $530 \mathrm{~nm}$ (yellow-green light). These colors were used in the examination of blood vessels of the eye. Illuminating the fundus with blue light stimulates all structures of the eye containing fluorescein, which fluoresces green-yellow light. 
Maximising the contrast between areas with fluorescein and without this colour is achieved through light filtration with wavelengths of $\lambda_{\mathrm{A}}$ and $\lambda_{\mathrm{E}}$, Fig. 2. The bandpass filter (B) leaves white light (A) in the spectrum with a wavelength of $\lambda_{\mathrm{CWL}} \in[465 \mathrm{~nm}, 490 \mathrm{~nm}]$, where $\mathrm{CWL}$ means center wavelength. The band-pass filter (D) passes through wavelengths of $\lambda_{\mathrm{CWL}} \in[520 \mathrm{~nm}, 530 \mathrm{~nm}]$. In reality the creation of such a system of filters is difficult.

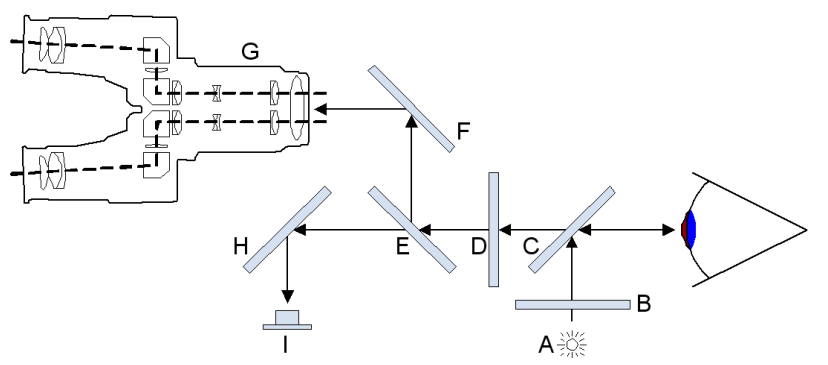

Fig. 2. System observation of fluorescence structures of the eye [17]: white light source (A); bandpass filter $\lambda_{\mathrm{CWL}} \in[465 \mathrm{~nm}, 490 \mathrm{~nm}](\mathrm{B})$; prism (C); bandpass filter $\lambda_{\mathrm{CWL}} \in[520 \mathrm{~nm}, 530 \mathrm{~nm}](\mathrm{D}) ; 50 \mathrm{R} / 50 \mathrm{~T}$ plate beam splitter $(\mathrm{E})$; reflecting mirror $(\mathrm{H}, \mathrm{F})$; CCD sensor $(\mathrm{I})$; eyepiece $(\mathrm{G})$.
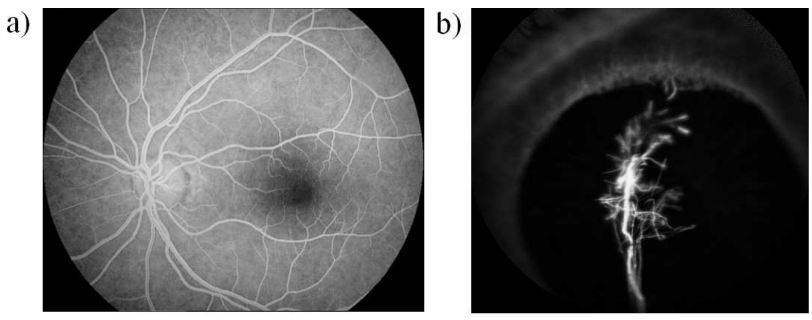

Fig. 3. Fluorescent angiography of blood vessels from

Fig. 1: retina (a), cornea (b).

The bandwidth of filters (B) and (D) in available devices partially overlap. This results in the formation of a phenomenon known as pseudofluorescence, which impedes the interpretation of test results.

The given technique is used mainly for testing chorioretinal circulation - retinal and retinal pathology. The experiments performed at the Warsaw Military Institute of Medicine showed that it is also possible to conduct blood vessels FA imaging of the cornea, Fig. 3b. However, this process takes place at a much higher dynamism than in the case of the retina. The fluorescence of the blood vessels of the cornea appears and disappears just as quickly. The average time in the experiments was about $3 \mathrm{~s}$.

An important limitation of the use of FA is the side effects associated with the administration of contrast. They include: yellowing of the skin, mucous membranes and urine as well as falsification of certain chemical tests with serum for several days after the test. From $2 \%$ to $4 \%$ of testees after $3060 \mathrm{~s}$ feel nauseous after administering the contrast, which can lead to vomiting. Other side effects that should be mentioned include urticaria, asthma symptoms, swelling of the larynx. Symptoms such as fainting, anaphylactic reaction to fluorescein, heart attack, cardiac arrest or lack of breathing are relatively rare.
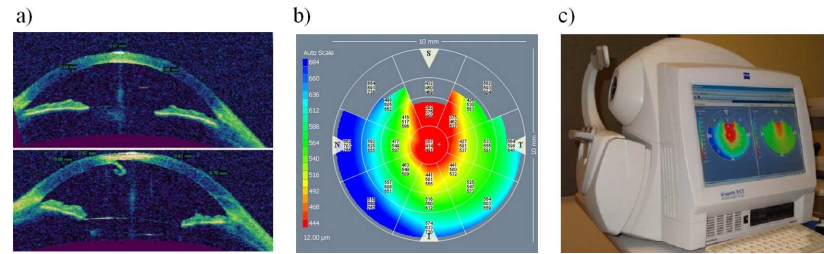

Fig. 4. OCT Visante: measuring the thickness of the corneal (a), pachymetry map (b), a view of the device $(c)$.

Another technique helpful in determining the degree of neovascularisation of the cornea is to measure its thickness, Fig. 4a. This study is justified due to the high degree of correlation between the neovascularisation of the cornea (usually mature vessels and their permeability) and the change of its thickness resulting from swelling. Corneal oedema can be observed by performing a test optical coherence tomography (OCT). During the study the pachymetry map is determined, Fig. 4b. For this purpose, the Visante OCT (OCTV) device was used in the experiments, Fig. 4c. The OCTV operates on the basis of the Michelson interferometer, Fig. 5 OCTV as the source of light (A) uses a superluminescent diode [18]. The LED emits light with a wavelength of $\lambda_{1} \in[820 \mathrm{~nm}, 830 \mathrm{~nm}]$ and $\lambda_{2}=1310 \mathrm{~nm}$, with a low coherence time and a high spatial coherence. The emitted light reaches the beam splitter $(\mathrm{C})$, where the reference beam is created and directed to the reference mirror (D) and studied beam is directed through a system of mirrors (E, F) onto the tested object $(\mathrm{K})$. The reflected beam of light from the object interferes with the reference beam. The beam resulting from light interference is directed onto a diffraction grating $(\mathrm{H})$. The diffraction grating forms the spectrum of light recorded by the camera $(\mathrm{J})$. This spectrum is processed and visualised by a computer system. Axial resolution in the OCT study (for OCTV $-18 \mu \mathrm{m}$ ) is conditioned by the scope of the scanning wave coherence. Lateral resolution (for OCTV $-60 \mu \mathrm{m}$ ) is linked to the number of scans forming the plane. The study used a pachymetric map (a map as thick as the cornea) defined by the OCTV. The pachymetric map is the result of measurements made in 8 lines 128 scans each with a frame scan size of $10 \mathrm{~mm}$ to $3 \mathrm{~mm}$. It is presented on the screen, Fig. $4 \mathrm{~b}$, in the form of a (image) colour map and in numerical form. Warm colors on the colour scale represent thinner areas of the cornea, while cool colours thicker areas. The result of the pachymetry is presented for ray ranges from $0 \mathrm{~mm}$ to $2 \mathrm{~mm}$, from $2 \mathrm{~mm}$ to $5 \mathrm{~mm}$, from $5 \mathrm{~mm}$ to $7 \mathrm{~mm}$ and from $7 \mathrm{~mm}$ to $10 \mathrm{~mm}$. The software allows expressing the measurement result of changes in corneal thickness in an absolute and relative scale. The assessment of the absolute deviation is based on the assumption that the highest on the colour scale 
(navy) defines the thickness of the cornea $l \geq 740 \mu \mathrm{m}$, while the lowest most color (orange) defines the thickness of the cornea $l \leq 340 \mu \mathrm{m}$. In the analysis of relative deviation the colors blue and orange indicate the thickest and thinnest areas of the cornea.

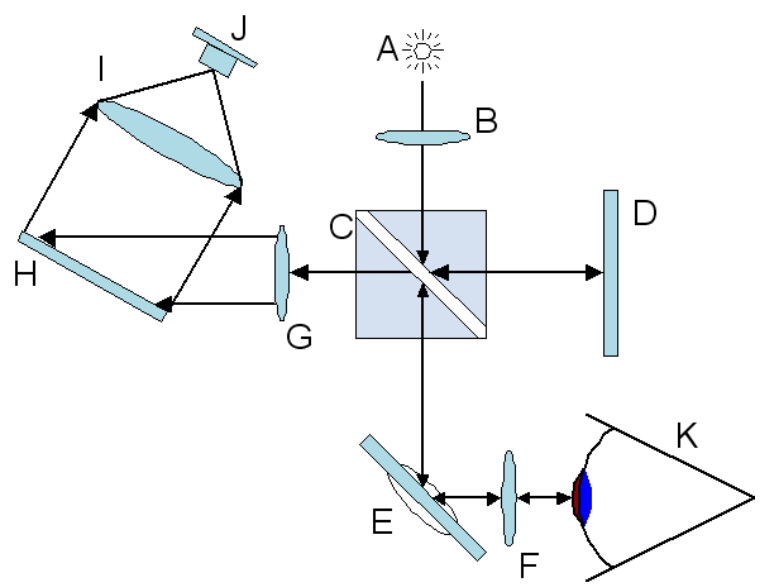

Fig. 5. Schematic diagram of Michelson interferometer [18].

Optical coherent tomography is a non-invasive test of the cornea providing high resolution images. This method was considered the "gold standard" in imaging the anterior segment of the eye. For this reason, and also due to high accuracy and repeatability of results, this method has been accepted as a reference method in the experiment (including angiography). The only restriction in its common application is the price of the device of over $\$ 65,000$ USD.

\section{Measurements of corneal neovascularisation with the use of image processing techniques}

The measurements of corneal neovascularisation was performed using digital image processing, Fig. 6 . The video source was a camera with a CCD image sensor mounted on a slit lamp, Fig. 1a. Photos at a resolution of $1024 \mathrm{px} \times 768 \mathrm{px}$ and a 24-bit colour depth were taken in a visible band with white light illuminating the cornea.

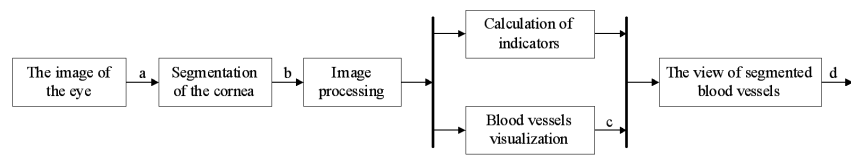

Fig. 6. Segmentation of blood vessels algorithm.

The proposed method of image segmentation has two stages. The first stage involves determining the location of the cornea on the image plane. The implementation of this operation is performed manually. Location of the cornea may be ultimately sought through the use of artificial intelligence algorithms [19] or determined by the algorithm given in [20]. Then the area of the cornea is determined indirectly by indicating the position of the iris. The second stage consists in image segmentation in order to determine the position of blood vessels of the cornea, Fig. 7. A colour image of the cornea $\mathrm{O}^{\mathrm{R}}$ is divided into constituent colors, images: $\mathrm{O}_{\mathrm{R}}, \mathrm{O}_{\mathrm{G}}$ and $\mathrm{O}_{\mathrm{B}}$. Further operations are performed on $\mathrm{O}_{R}$ and $\mathrm{O}_{\mathrm{G}} \cdot \mathrm{O}_{\mathrm{B}}$ is used in the process of visualisation of the map of blood vessels. $\mathrm{O}_{\mathrm{R}}$ and $\mathrm{O}_{\mathrm{G}}$ are processed to improve contrast. For this purpose, the imadjust function in Matlab was used. Then the $\mathrm{R}$ channel is subjected to the blur operation. The $\mathrm{O}_{\mathrm{R}}$ blur was performed with function: imfilter (R, PSF, "circ", "conv"), where the PSF = fspecial ("average", [10 10]). The achieved image $\mathrm{O}_{\mathrm{R}}$ contains virtually no blood vessels. Afterwards $\mathrm{O}_{\mathrm{G}}$ is subtracted from image $\mathrm{O}_{R}$. The resulting $\mathrm{O}_{R G}=\mathrm{O}_{R}-\mathrm{O}_{G}$ is subject to correction using the average pixel value and mean determined from $\mathrm{O}_{\mathrm{RG}}$ as well as multipliers $n=1.5$ and $k=5$. The values of the multipliers were experimentally chosen. The new values of $W^{\prime}\left(p_{i j}\right)$ pixels of $p_{i j}$ image are determined from Eq. (1):

$$
W^{\prime}\left(p_{i j}\right)= \begin{cases}0 & \text { for } W\left(p_{i j}\right)<n \cdot \text { mean }, \\ k W\left(p_{i j}\right) & \text { for } n \cdot \text { mean } \leq k W\left(p_{i j}\right) \leq 255, \\ 255 & \text { for } k \cdot W\left(p_{i j}\right)>255,\end{cases}
$$$$
p_{i j}=\mathrm{O}^{\mathrm{R}}(i, j) \text {; }
$$

$W\left(p_{i j}\right)$ - pixel color value. Subsequently, the $\mathrm{O}_{\mathrm{RG}}$ image is multiplied, pixel by pixel, with the image $\mathrm{O}^{\mathrm{R}}$ obtaining $\mathrm{O}_{\mathrm{RG}}^{\mathrm{R}}$. In $\mathrm{O}_{\mathrm{RG}}^{\mathrm{R}}$ the area beyond the surface of the cornea takes on the colour black. The corneal area not containing any blood vessels is white. The cornea areas containing blood vessels are marked in $\operatorname{red}(\mathrm{R}=255$, $\mathrm{G}=0, \mathrm{~B}=0)$ and yellow $(\mathrm{R}=255, \mathrm{G}=255, \mathrm{~B}=0)$. Colour unification of image $\mathrm{O}_{\mathrm{RG}}^{\mathrm{R}}$ was achieved using correction of pixel values of the red channel. The colours of image $\mathrm{O}_{\mathrm{RG}}^{\mathrm{R}}$ are adjusted in accordance with principles: if the RGB channels of image $O_{\mathrm{RG}}^{R}$ are equal $(255,255,0)$ then the value in the channel $G$ is set to 0 , if the value of the RGB channels are different from $(255,0,0)$ then they are set to $(0,0,0)$. After completing the correction procedure channel $R$ of the improved image $\mathrm{O}_{R G}^{R}$ is combined with channels $\mathrm{G}$ and $\mathrm{B}$ of image $\mathrm{O}^{\mathrm{R}}$. This way the source image is reproduced simultaneously emphasizing the locations of blood vessels. The last step is to determine the number of pixels in groups of pixels of selected colours (black, white, red). These values indicate the quantitative as well as the percentage of corneal coverage by the blood vessels formed during the process of neovascularisation of the cornea. The partial results of processing the exemplary image of the cornea in points (a)-(o) of the algorithm are shown in Fig. 6 and Fig. 7 is included in Fig. 8.

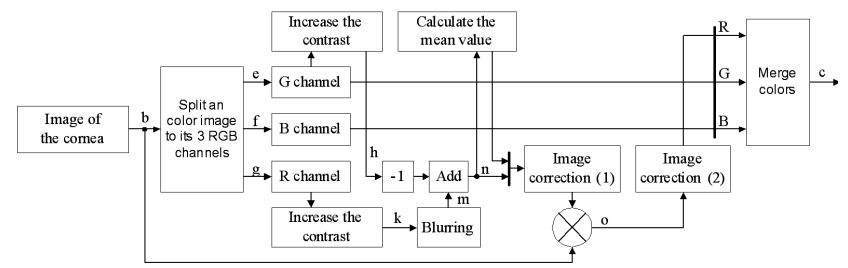

Fig. 7. Image processing block. 


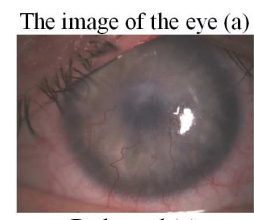

R channel $(\mathrm{g})$

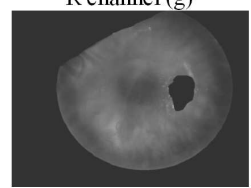

R channel inc. contr. (k)

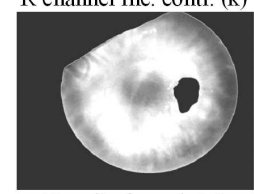

Result of $\mathrm{m}-\mathrm{h}(\mathrm{n})$

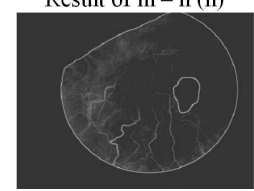

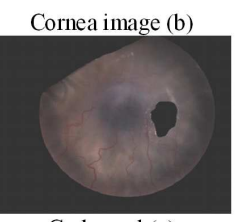

G channel (e)

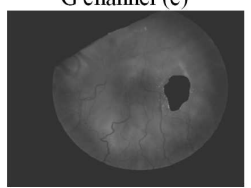

G channel inc. contr. (h)

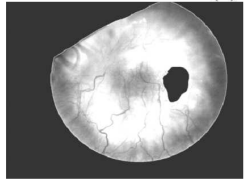

Blood vessels (o)

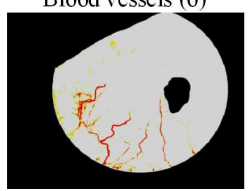

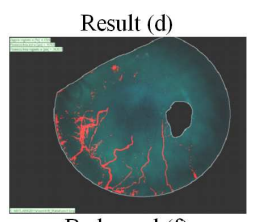

B channel (f)

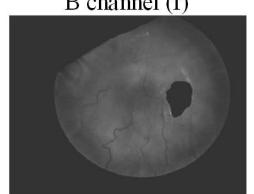

Blurred R channel (m)

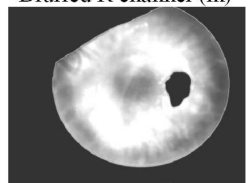

Corrected blood vessels (c)

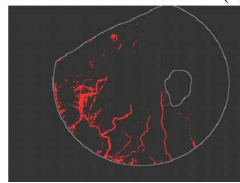

Fig. 8. Results of the algorithm from Fig. 6, Fig. 7 in points a-o during image processing of the cornea.

The area of the blood vessels in the presented example equaled $15763 \mathrm{px}$, and the area of the cornea $379600 \mathrm{px}$. The calculated corneal neovascularisation equaled $4.15 \%$ of its surface.

\section{Results of research}

The presented technique of image processing has been studied on simulated and real images. For artificially produced images $100 \%$ of the simulated blood vessels area was defined. For real images no alternative techniques were available for determining the surface of the blood vessels of the cornea. However, the conformity of determining their position was achieved when compared to the results of angiography and OCTV testing. An example of positioning the blood vessels on the image taken with a slit lamp and the image obtained during angiography of the cornea is shown in Fig. 9. Determined areas where blood vessels appear were outlined.
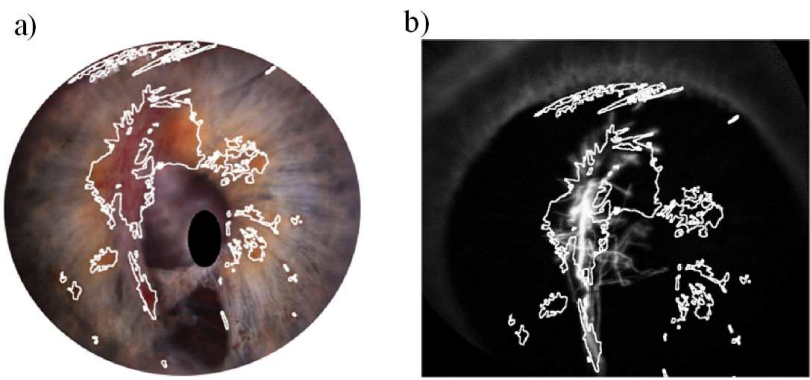

Fig. 9. Processing results (a), a composition with the result of the angiography (b).

Determined areas cover the same area as the blood vessels indicated during angiography. Other examples of actions of the proposed method were presented in Fig. 10. a)

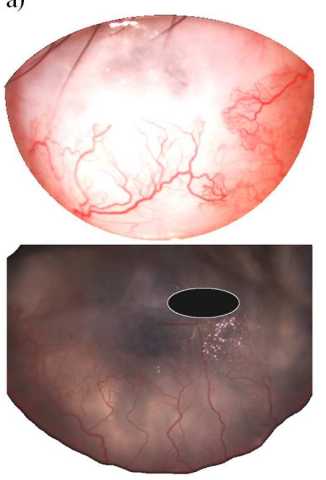

b)

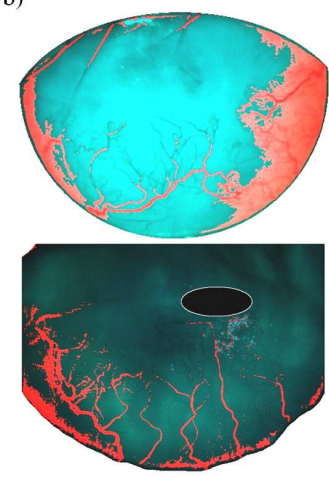

Fig. 10. An example of the results obtained by proposed measurement technique: view of the cornea (a) processing result (b).

\section{Conclusions}

The study shows that the utilisation of the developed image processing technique to measure the corneal neovascularisation is very promising. The obtained results show that it is possible to conduct the segmentation of blood vessels of the cornea. The results obtained during digital imaging coincide with the results of angiography. Simultaneously, the technique proposed in the study is non-invasive, safe, short and sensitive. The developed technique confirmed in the course of the study that it can considerably help to objectively assess used methods of treatment.

\section{Acknowledgments}

The Matlab Software was co-financed by the Polish Ministry of Science grant no. 3348/IA-LAN/2012. The work presented in the paper was made under project No. 5O99/E-592/M/2012 co-financed by the Military Institute of Medicine (D. Białas and M. Rękas) as well as Military University of Technology (K. Murawski).

The work was presented at the 8th Conference Integrated Optics - Sensors, Sensing Structures and Methods, IOS'2014 sponsored by Polish Academy of Sciences. The conference was organized by the Committee of Electronics and Telecommunication at the Polish Academy of Sciences in cooperation with the Upper Silesian Division of the Polish Acoustical Society and Photonic Society of Poland, as well as the Department of Optoelectronics at the Silesian University of Technology.

\section{References}

[1] R.C. Arffa, Duane's Ophthalmology on CD-ROM, Lippincott Williams \& Wilkins Publ., Vol. 64, 2006.

[2] Y.S. Rabinowitz, Duane's Ophthalmology on CDROM, Lippincott Williams \& Wilkins Publ., Vol. 65, 2006.

[3] G.K. Klintworth, Corneal Angiogenesis: A Comprehensive Critical Review, Springer-Verlag, New York 1991, pp. 1; 13. 
[4] M.E. Gegúndez-Arias, A. Aquino, J.M. Bravo, D. Marín, Proc. IEEE Trans. Med. Imag. 31, 2 (2012).

[5] H. Narasimha-Iyer, J.M. Beach, B. Khoobehi, B. Roysam, Proc. IEEE Trans. Biomed. Eng. 54, 1427 (2007).

[6] M. Usman Akram, S. Farrukh Aneeque, A. Atzaz, S.A. Khan, in: Proc. Int. Conf. on Digital Image Processing, Bangkok 2009, p. 34.

[7] M. Ben Abdallah, J. Malek, K. Krissian, R. Tourki, in: Proc. 8th Int. Multi-Conf. on Systems, Sousse 2011, p. 1.

[8] K. Murawski, R. Różycki, P. Murawski, A. Matyja, M. Rękas, Acta Phys. Pol. A 124, 517 (2013).

[9] P. Versura, V. Profazio, E.C. Campos, Acta Ophthalmol. 88, 246 (2010).

[10] K. Różanowski, K. Murawski, Acta Phys. Pol. A 124, 558 (2013).

[11] K. Różanowski, K. Murawski, Acta Phys. Pol. A 124, 874 (2013).

[12] K. Murawski, K. Różanowski, Acta Phys. Pol. A 124, 509, (2013).
[13] K. Murawski, in: Proc. 15th Int. Conf. on Methods and Models in Automation and Robotics (MMAR), 2010 , p. 1.

[14] K. Murawski, Przegląd Elektrotechniczny 9, 184 (2010) (in Polish).

[15] M. Dastjerdi, K. Al-Arfaj, N. Nallasamy, P. Hamrah, U. Jurkunas, R. Pineda, D. Pavan-Langston, R. Dana, Arch. Ophthalmol. 127, 381 (2009).

[16] D.H. Orth, J.S. Kelley, J.W. Berkow, R.W. Flower, Fluoresce in Angiography and Indocyanine, Vol. 1, Górnicki Wydawnictwo Medyczne, Wrocław 2003, p. 1 (in Polish).

[17] J. Kałużny, A. Mierzejewski, S. Milewski, Research Angiographic Fundus, Vol. 1, 1998, p. 1 (in Polish).

[18] E. Wylęgała, A. Nowińska, S. Teper, Optical Coherence Tomography, Vol. 1, 2010, p. 9 (in Polish).

[19] K. Murawski, T. Arciszewski, K. De Jong, Eng. Comput. 16, 275 (2000).

[20] J. Daugman, Proc. IEEE Trans. Circuits Syst. Video Technol. 14, 21 (2004). 